\title{
Spin mixing in colliding spinor condensates: formation of an ef- fective barrier
}

\author{
M. Guilleumas ${ }^{1}$, B. Juliá-Díaz ${ }^{1}$, J. Mur-Petit ${ }^{2}$, A. Polls ${ }^{1}$ \\ Departament d'Estructura $i$ Constituents de la Matèria, Universitat de Barcelona, E-08028 Barcelona, Spain \\ Department of Physics and Astronomy, University College London, London WC1E 6BT, United Kingdom.
}

PACS $03.75 . \mathrm{Mn}$ - Multicomponent condensates; spinor condensates
PACS 03.75. Kk - $\begin{aligned} \text { Dynamic properties of condensates; collective and hydrodynamic excitations, } \\ \text { superfluid flow }\end{aligned}$
PACS 03.75. Lm - Tunneling, Josephson effect, Bose-Einstein condensates in periodic potentials,
solitons, vortices, and topological excitations

\begin{abstract}
The dynamics of $F=1$ spinor condensates initially prepared in a double-well potential is studied in the mean field approach. It is shown that a small seed of $m=0$ atoms on a system with initially well separated $m=1$ and $m=-1$ condensates has a dramatic effect on their mixing dynamics, acting as an effective barrier for a remarkably long time. We show that this effect is due to the spinor character of the system, and provides an observable example of the interplay between the internal spin dynamics and the macroscopic evolution of the magnetization in a spinor Bose-Einstein condensate.
\end{abstract}

Introduction. - Ultracold atoms trapped by optical means are suitable systems to address a broad range of problems related to magnetic ordering and dynamics. Soon after the pioneering experiments with $F=1{ }^{23} \mathrm{Na}$ at MIT [1] a number of groups have managed to observe spinor dynamics in a variety of conditions, e.g. in a quasi$1 \mathrm{D}$ system [2] or a $2 \mathrm{D}$ trap [3], thus being able to experimentally address many of the questions posed by theoreticians $[4,5]$.

Among the latter, the existing relation between the internal dynamics and the spatial ordering in Bose-Einstein Condensates (BEC) provides a beautiful example of how the microscopic interactions shape the macroscopic properties of the BEC. As discussed recently in [6-8] one such example is the formation of magnetic domains in $F=1$ spinor systems (see also [9]). In Ref. [8] the dynamical evolution of a 1D confined BEC with non-equilibrium initial populations in the spin components was studied in the mean field approach. As the main emphasis of that study was on domain formation, the initial configurations considered were always such that there was no relative motion between the center of mass of the different Zeeman components.

In this paper we consider the complementary case of initial conditions where the spin components are spatially well separated inside a harmonic trap, ensuring a cer- tain amount of relative center of mass motion between them. The subject of collisions between scalar BECs has been addressed in several experiments, revealing interesting features of collective modes [10] and even realizing an atom analogue of the Hanbury Brown-Twiss effect of optics [11]. Other experimental studies on the dynamics of spinor BECs [12], as well as binary mixtures [13,14], have focused on the evolution of the spatial distribution of the different spin components in the system. Specially interesting is the observation by Hall et al. [13] of a fast convergence to a configuration with spin-segregation in the case of two interacting initially overlapping BECs of ${ }^{87} \mathrm{Rb}$ in different hyperfine states.

The setup considered here can be seen as a low energy confined version of those in Ref. [15], where collisions of unconfined BECs are analyzed. In the present work the system is confined and thus no atoms escape the trap.

The paper is organized as follows. First the simplest extension of $[8,16]$ is considered, namely the formation of magnetic domains in a trapped $F=1 \mathrm{BEC}$ with no relative center of mass momenta between the Zeeman components even when the center of mass momentum of the system is not zero. Under these premises a full decoupling of the Kohn mode [17], dipolar oscillation in the confining trap, and the internal spin dynamics is found.

In the second step we introduce initial spatial separa- 
tion between the different components, e.g. $m=-1$ on the right side of the trap and $m=+1$ on the left side, which are initially kept separated by means of a gaussian barrier. Switching off the barrier at $t=0$ the condensates collide and the time evolution of the different populations is analyzed.

With these conditions we find a remarkable effect. Namely that on $F=1$ BECs with initially separated $m=-1$ and $m=+1$ components, a small amount of $m=0$ component produces a barrier-like effect which prevents the mixing of the $m= \pm 1$ components for a long time. We further show that this effect is characteristic of spinor BECs, in contrast e.g. to binary mixtures, and occurs in a time scale reachable in current experiments with ultracold atoms.

Description of the system. - In the mean-field approach, the $F=1$ spinor condensate is described by a vector order parameter $\Psi$ whose components $\psi_{m}$ correspond to the wave function of each magnetic sublevel $|F=1, m\rangle \equiv|m\rangle$ with $m=1,0,-1$. In absence of an external magnetic field and at zero temperature the spin dynamics of this system confined in an external potential, $V_{\text {ext }}$, is described by the following coupled equations for the spin components $[4,8]$ :

$$
\begin{aligned}
i \hbar \partial \psi_{ \pm 1} / \partial t= & {\left[\mathcal{H}_{s}+c_{2}\left(n_{ \pm 1}+n_{0}-n_{\mp 1}\right)\right] \psi_{ \pm 1} } \\
& +c_{2} \psi_{0}^{2} \psi_{\mp 1}^{*}, \\
i \hbar \partial \psi_{0} / \partial t= & {\left[\mathcal{H}_{s}+c_{2}\left(n_{1}+n_{-1}\right)\right] \psi_{0} } \\
& +c_{2} 2 \psi_{1} \psi_{0}^{*} \psi_{-1},
\end{aligned}
$$

with $\mathcal{H}_{s}=-\hbar^{2} /(2 M) \nabla^{2}+V_{\text {ext }}+c_{0} n$ being the spinindependent part of the Hamiltonian. The density of the $m$-th component is given by $n_{m}(\mathbf{r})=\left|\psi_{m}(\mathbf{r})\right|^{2}$, while $n(\mathbf{r})=\sum_{m}\left|\psi_{m}(\mathbf{r})\right|^{2}$ is the total density normalized to the total number of atoms $N$. The population of each hyperfine state is $N_{m}=\int d \mathbf{r}\left|\psi_{m}(\mathbf{r})\right|^{2}$. Defining the relative populations $\lambda_{m}=N_{m} / N$, it follows that $\lambda_{1}+\lambda_{0}+\lambda_{-1}=1$ and the magnetization of the system is $\mathcal{M}=\lambda_{1}-\lambda_{-1}$. The total number of atoms and the magnetization are both conserved quantities [8]. The couplings are $c_{0}=$ $4 \pi \hbar^{2}\left(a_{0}+2 a_{2}\right) /(3 M)$ and $c_{2}=4 \pi \hbar^{2}\left(a_{2}-a_{0}\right) /(3 M)$, where $M$ is the atomic mass and $a_{0}, a_{2}$ are the scattering lengths describing binary elastic collisions in the channels of total spin 0 and 2 , respectively. The interatomic interactions permit the transfer of population between the different Zeeman components by processes that conserve the total spin, $|0\rangle+|0\rangle \leftrightarrow|+1\rangle+|-1\rangle$.

Preparation of the system. - We consider $N=$ 20000 atoms of spin- ${ }^{87} \mathrm{Rb}$ in a highly elongated trap with $\omega_{\perp}=2 \pi \times 891 \mathrm{~Hz}$ and $\omega_{z}=2 \pi \times 21 \mathrm{~Hz}$ [2]. The scattering lengths are $a_{0}=101.9 a_{B}$ and $a_{2}=100.4 a_{B}$ corresponding to a ferromagnetic behavior, $c_{2}<0$ [4]. Since $\omega_{\perp} \gg \omega_{z}$ the dynamics takes place along the axial direction and the equations of motion become one-dimensional for the longitudinal wave functions $\psi_{m}(z)$ by rescaling the coupling constants $c_{0}$ and $c_{2}$ by a factor $1 /\left(2 \pi a_{\perp}^{2}\right)$, with $a_{\perp}$ the transverse oscillator length $[16,18]$. Overlapped with the harmonic potential we consider a gaussian barrier which separates the system in two symmetric wells (labeled as $R$ and $L$, corresponding to the right and left side respectively). The resulting confining potential is the same for the three Zeeman components, and reads:

$$
V_{\text {ext }}=\frac{m}{2} \omega_{z}^{2} z^{2}+A \exp \left(-z^{2} / \sigma^{2}\right) .
$$

Given an initial configuration of the system (the relative population of spin components inside each potential well: $\lambda_{m}^{j}$, with $m=1,0,-1, j=L, R$ and $\left.\lambda_{m}=\lambda_{m}^{L}+\lambda_{m}^{R}\right)$, the initial state of the system is prepared as follows: (i) First the ground-state wave function of the scalar condensate is calculated from $\mathcal{H}_{s}$ on the whole spatial domain. The presence of a wide and large enough barrier at the center of the trap ensures no overlap between the wave function at each side of the barrier. Notice that due to the presence of the initial barrier the two wells are not parabolic and the wave function in each well is strongly non-parabolic, see inset of Fig 2, (ii) Afterwards, the wave function of each magnetic sublevel inside each well is obtained by normalizing the scalar wave function to the desired initial relative population $\lambda_{m}^{j}$ in that well [16]. (iii) At $t=0$ the potential barrier is instantaneously switched off and the spinor system is allowed to evolve inside the harmonic 1D-potential. The dynamics follows by solving the system of coupled equations (12). Our numerical procedure for the time evolution combines the split operator method with the fast Fourier transform to treat the kinetic terms and a fourth-order Runge-Kutta method for the remaining terms of the dynamical equations [8].

Scalar condensate. - In order to understand the spinor-driven effects on the condensate dynamics, let us first address a spin-polarized condensate of $m=-1$ atoms all of them localized on the right side of the trap: $\lambda_{m}^{L}=0$ (for all $m$ ), $\lambda_{1}^{R}=\lambda_{0}^{R}=0$ and $\lambda_{-1}^{R}=1$. Since all the atoms are in the same hyperfine state this case is equivalent to having a scalar condensate. At $t=0$ the gaussian barrier is switched off and the system evolves inside the harmonic trap bouncing from right to left in the well-known Kohn mode [17].

In Fig. 1 we depict the fraction of atoms in the left side of the trap as a function of time (solid line). For comparison, the dipole oscillation of the ground state of a displaced harmonic trap is also displayed (dashed line). The Kohn mode is clearly identified by the frequency of the population oscillations being equal to the axial frequency of the trap $\omega_{z}$. Further, as our initial configuration is not the ground state of the harmonic trap, the Kohn oscillation is convoluted with other frequencies associated with the deformation of the density profile that bounces inside the trap.

Component driven dynamics. - We proceed now to introduce spin dynamics in the system. To this end, we analyze the time evolution of a system with all three 


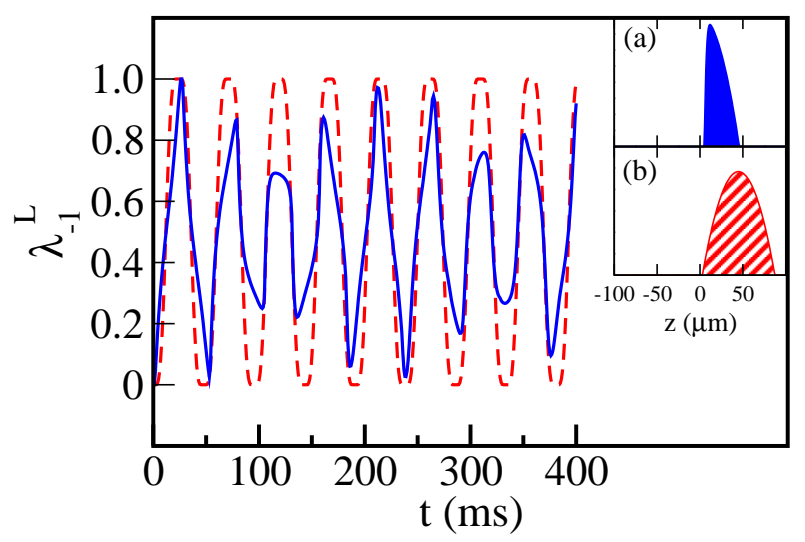

Fig. 1: Fraction of atoms on the left side of the trap as a function of time for two different initial states of a scalar BEC (sketched in the inset): (a) harmonic trap with a gaussian barrier (solid line) and (b) ground state of a displaced harmonic trap (dashed).

Zeeman components populated, and the atoms initially in the right side of the trap.

If we start with $\lambda_{ \pm 1}^{R}=25 \%$ and $\lambda_{0}^{R}=50 \%$ (and $\lambda_{m}^{L}=0 \forall m$ ), which corresponds to the ground-state configuration in spin space [8], we observe no transfer of population among the different components, while the total density follows the same behaviour as the solid line in Fig. 1 (Kohn mode). Moreover, if we start from a spin configuration away from the ground state, the total density still follows a dipole-like oscillation, on top of which a transfer of population happens identical to that occurring in the case of a system at rest in a simple harmonic trap $[8,19]$. In other words, the center-of-mass motion and the internal (spin) dynamics are fully decoupled, as expected for the dipole mode in a harmonic trap [20]. We remark that the time evolution of $\lambda_{m}(t)$ is exactly the same as in the system at rest. The relative populations oscillate around the corresponding equilibrium values at a given magnetization (e.g., $\lambda_{0}=50 \%, \lambda_{ \pm 1}=25 \%$ for $\mathcal{M}=0)$, as was already pointed out in Ref. [16].

We have seen how the presence of internal spin dynamics does not affect the evolution of the total density $n(z, t)$ of the system when all the atoms have the same initial spatial distribution. Now we will illustrate how the exact initial conditions of the system (spin configuration and spatial distribution thereof) can have a strong effect on the internal and the spatial dynamics, showing genuine effects associated to the spinor nature of the condensate.

Let us consider the case where initially all $m=1$ ( $m=-1$ ) atoms are located on the left (right) side of the trap, with a vanishing overlap between the two condensates $\left(\int_{-\delta}^{+\delta} \mathrm{d} z\left[n_{-1}(z)+n_{+1}(z)\right]=0\right.$ for $\left.\delta=4 \mu \mathrm{m}\right)$. A small amount of atoms with $m=0$ is added on both sides, $\lambda_{0}^{L}=\lambda_{0}^{R}=1 \%$, together with $\lambda_{1}^{L}=\lambda_{-1}^{R}=49 \%$ and $\lambda_{-1}^{L}=\lambda_{1}^{R}=0$ (see inset of Fig. 2). This small admixture of $m=0$ allows the exchange of atoms between spin com-

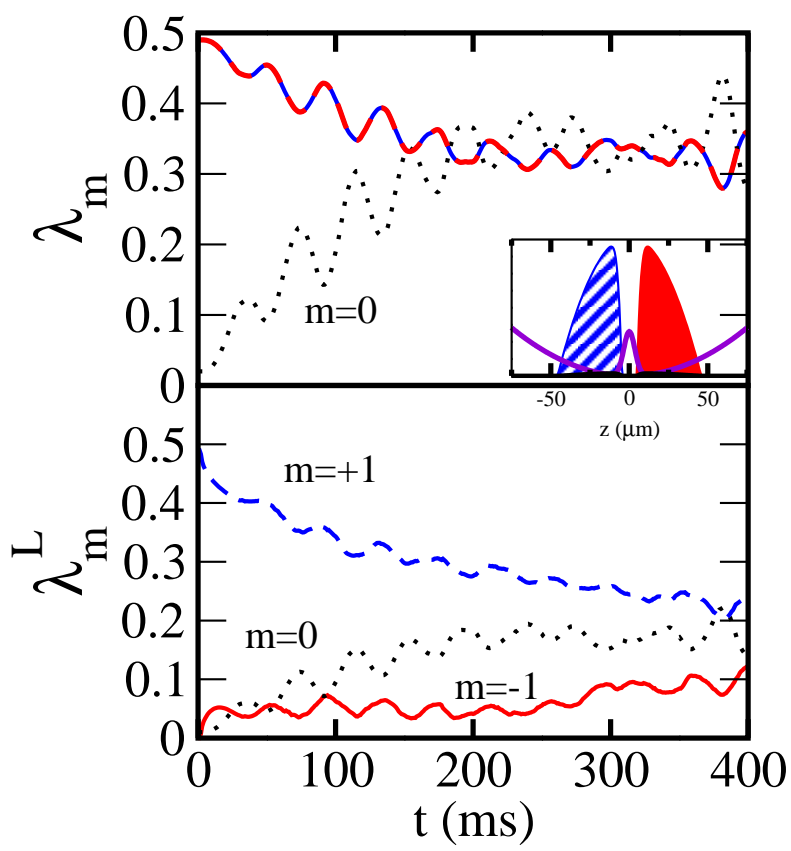

Fig. 2: Time dependence of the total (top) and fraction on the left side (bottom) relative populations of the various Zeeman components of a spinor condensate, for the initial conditions $\lambda_{1}^{L}=\lambda_{-1}^{R}=49 \%, \lambda_{0}^{R}=\lambda_{0}^{L}=1 \%$. Solid, dotted and dashed correspond to $m=-1, m=0$ and $m=+1$ respectively. Notice that the lines for $m= \pm 1$ in the top panel are indistinguishable.

ponents: $|0\rangle+|0\rangle \leftrightarrow|+1\rangle+|-1\rangle$ within the approach of Eqs. (12). Then, the population of each sublevel changes with time but due to the symmetry of the equations and the initial conditions we have that for any $t, \lambda_{1}(t)=\lambda_{-1}(t)$ (see Fig. 2).

The resulting dynamics after switching off the trap is as follows. At short times, $m=0$ atoms are created at the center of the trap where the overlap between $m= \pm 1$ components is larger [cf. Eq. (2)]. Here the $m= \pm 1$ components fuse together giving rise to $m=0$ atoms with a conversion rate proportional to $c_{2}$. This can be seen in the lower panel of Fig. 2, where we present the relative populations on the left side of the trap as a function of time. We observe after $\sim 5 \mathrm{~ms}$ a temporary saturation of the number of $m=-1$ atoms on the left side for up to $350 \mathrm{~ms}$, while the $m=+1$ component keeps losing population, which is transferred to the $m=0$ Zeeman state. The oscillations seen in all $\lambda_{m}^{j}$ in Fig. 2 are reminiscent of the Kohn mode discussed above, and have a dominant frequency close to that of the harmonic trap $\left(\omega_{z}\right)$.

The long time behaviour observed in Fig. 2 is determined by the initial conditions that correspond to a highly excited configuration. After the clouds move towards each other and collide, the initial collision energy is distributed among internal degrees of freedom, thus acting as an effective temperature. Therefore the populations perform damped oscillations around the equipartition configuration $[16]$. 


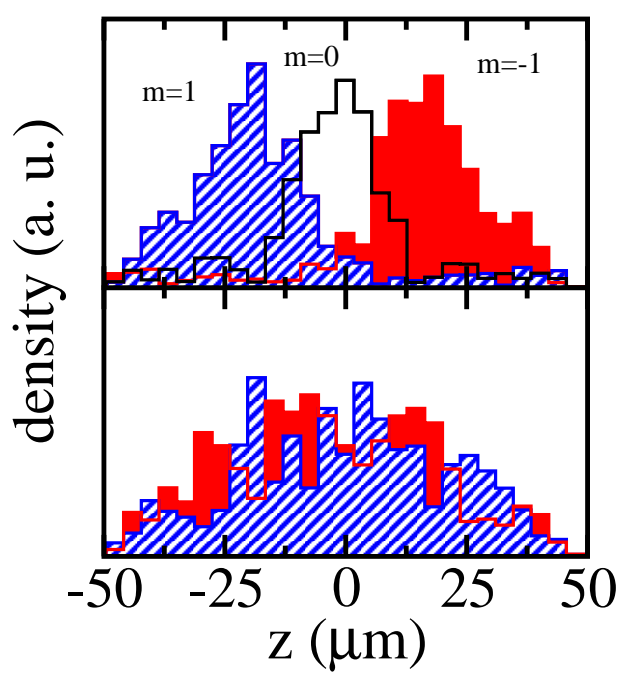

Fig. 3: Density distribution of atoms in the trap, at time $t=$ $115 \mathrm{~ms}$ with $\lambda_{0}(t=0)=2 \%$ (top) and for the case of $\lambda_{0}(t=$ $0)=0$ (bottom). Solid, empty and dashed correspond to $m=$ $-1, m=0$ and $m=+1$. A $\sim 4 \mu \mathrm{m}$ spatial binning has been employed to generate the plot.

To further understand the effect of the $m=0$ component, we show in the upper panel of Fig. 3 the density profile of the various Zeeman components at $t=115 \mathrm{~ms}$, well into the time evolution of the system. We observe the presence of $m=0$ atoms located at the center of the trap, with $m=1(m=-1)$ atoms remaining to its left (right). In summary, the initial $m= \pm 1$ components are steadily converted into $m=0$ atoms which stay mostly at the center of the trap, and act as an effective barrier that prevents the spatial mixing of the surviving $m= \pm 1$ components. These stay mostly on their original side of the trap for several hundreds of miliseconds, with only a small fraction of atoms crossing over to the other side. The long lifetime that the $m=0$ barrier shows in this case is to be contrasted with the much faster spin dynamics seen previously, where the typical timescale for population transfer between initially overlapping Zeeman states was $\sim 100 \mathrm{~ms}$ [8]. It also contrasts with the spin-segregation time scale observed in binary mixtures of ${ }^{87} \mathrm{Rb}$ [13], as will be addressed below. Interestingly, qualitatively similar results have been obtained in an antiferromagnetic system simulated by changing the sign of $c_{2}\left(c_{2}>0\right)$.

We have also observed that even a very small $m=0$ seed in one side $(0.02 \%)$ has the same effect of acting as a barrier and stops the mixing of the $m= \pm 1$ atoms. However, as expected, different $\lambda_{0}(t=0)$ result in different transient behaviours. In particular, the larger the $m=0$ seed the longer the time necessary to have a mixture of the other Zeeman components with similar concentrations in both sides. Nevertheless, the long time $(\sim 400 \mathrm{~ms})$ configuration is roughly the same.

To emphasize the spinorial origin of this barrier effect we compare with the mixing process of two interacting BECs.

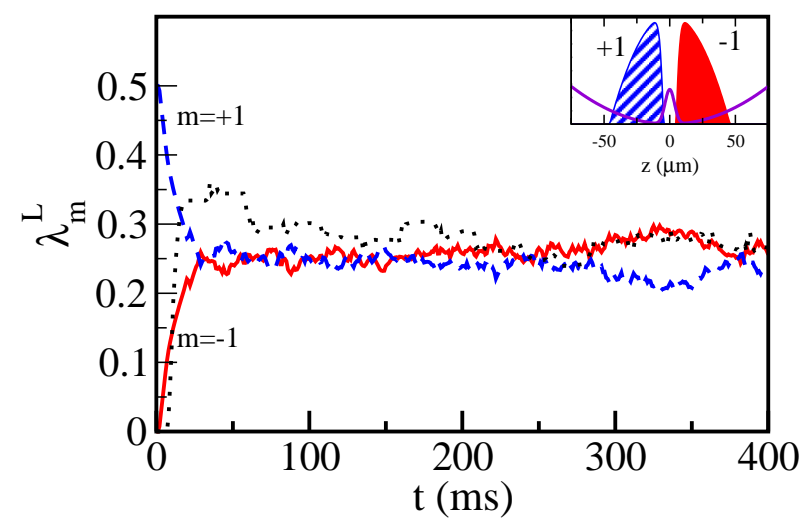

Fig. 4: Fraction of atoms in the $m=-1$ (solid) and $m=+1$ (dashed) Zeeman component on the left side of the trap, as a function of time for a binary mixture. The inset shows the initial spatial distribution together with the confining potential before switching off the $\sigma=5 \mu \mathrm{m}$ gaussian barrier. The dotted line stands for the time evolution of $\lambda_{-1}(t)$ for an initial configuration given by $\sigma=40 \mu \mathrm{m}$.

Such binary mixtures have been realized experimentally by coupling two Zeeman states via a two-photon drive. For example, mixtures of ${ }^{87} \mathrm{Rb}$ in the hyperfine states $\mid F=$ $2, m=2\rangle$ and $|2,1\rangle[10]$ as well as $|1,-1\rangle$ and $|2,1\rangle[13,14]$ have been both produced. In the mean-field framework their dynamics can be easily simulated with the coupled differential equations (112) by taking a zero amount of $m=$ 0 atoms.

The preparation of the system follows the same steps as above, setting now the initial conditions as $\lambda_{+1}^{L}=\lambda_{-1}^{R}=$ $50 \%$ and $\lambda_{+1}^{R}=\lambda_{-1}^{L}=0$. The barrier is switched off at $t=0$ and the two condensates move inside the harmonic trap towards each other, colliding after $\sim 10 \mathrm{~ms}$. As can be seen in Fig. 4, at this time a sizeable amount of atoms of each component has already reached the other side of the trap, and rapidly mixes with the other component. As expected, $\lambda_{-1}^{L}$ grows initially with a similar rate as it did in Fig. 2. but departs from that behaviour after times $\sim 5 \mathrm{~ms}$, due to atoms coming from the other side of the trap. There is also a similar decrease of $\lambda_{+1}^{L}$ since an equal amount of $m=1$ atoms goes to the right. Around $t=25 \mathrm{~ms}$ the two components are mixed and there is almost the same number of $m= \pm 1$ atoms in each side of the trap, i.e. there is a fast and complete mixing of both components, as shown in the lower panel of Fig. 3

If the width of the initial gaussian barrier is increased, the initial collision energy is larger, and thus the number of $m=-1$ atoms reaching the left side is also larger initially. However, it reaches the same asymptotic value as before (cf. dotted line in Fig. (4). Therefore, we conclude that the transition to a steady state is much slower in the spinor case than it is for a binary mixture (cf. Figs. 2 and 4 ), which qualitatively agrees with the observations in $[12,13]$.

Conclusions. - In summary, we have studied the interplay between the internal spin dynamics and the spatial 
evolution of a spin- 1 condensate in a confining potential. We have described the effect produced by the presence of $m=0$ atoms in a system initially prepared with two spatially separated spin components $m=+1$ and $m=-1$. An initial small population of the $m=0$ component drastically affects the mixing time of the system as compared to a binary mixture, producing a barrier-like behaviour which keeps the other two components from mixing for times up to $\sim 350 \mathrm{~ms}$ which is a timescale on which experimental observations should be feasible. We have also demonstrated the genuine spinor character of this effect, and that it does not appear in scalar condensates or binary mixtures. The population transfer term of Eqs (12) plays a key role in the generation of the effective barrier.

The seed of $m=0$ atoms can be a residual of the preparation of the initial state in an experiment. A similar effect would also be produced in an initially pure, $|+1\rangle|-1\rangle$ mixture by two-body spin-flip processes $|+1\rangle+|-1\rangle \rightarrow|0\rangle+|0\rangle$ at the initial stages of the evolution. Therefore, the presence of the effective barrier preventing the mixing of spin components appears as a general phenomenon in spinor BECs prepared with spatially separated Zeeman components.

In the future, we will address more general examples of interplay of spin and spatial degrees of freedom in spinor Josephson junctions whose barriers have a more complex time dependence, and can distinguish between different hyperfine components [21]. We note also that more exotic spatial orderings and dynamics are expected to occur in optical lattices, where oscillation in the populations of two different Zeeman components have already been reported [22].

$$
* * *
$$

It is a pleasure to acknowledge $\mathrm{K}$. Bongs, M. Lewenstein and L. Pitaevskii for a careful reading of an earlier version of the manuscript and also K. Sengstock for useful discussions. B.J-D. is supported by a 'Juan de la Cierva' contract, MEC/Spain. J.M.-P. acknowledges support from EPSRC (UK) and QUDEDIS (ESF). This work is also partially supported by Grants No. FIS2005-03142 and FIS2005-01414 from MEC (Spain) and 2005SGR-00343 from Generalitat de Catalunya.

\section{REFERENCES}

[1] J. Stenger, S. Inouye, D. M. Stamper-Kurn, H. J. Miesner, A. P. Chikkatur, and W. Ketterle, Nature 396, 345 (1998).

[2] H. Schmaljohann, M. Erhard, J. Kronjäger, M. Kottke, S. van Staa, L. Cacciapuoti, J. J. Arlt, K. Bongs, and K. Sengstock, Phys. Rev. Lett. 92, 040402 (2004).

[3] M.-S. Chang, C. D. Hamley, M. D. Barrett, J. A. Sauer, K. M. Fortier, W. Zhang, L. You, and M. S. Chapman, Phys. Rev. Lett. 92, 140403 (2004).

[4] T.L. Ho, Phys. Rev. Lett. 81, 742 (1998). C.K. Law, H. Pu, and N.P. Bigelow, ibid. 81, 5257 (1998). M. Koashi and M.
Ueda, ibid. 84, 1066 (2000). T. Ohmi and K. Machida, J. Phys. Soc. Japan 67, 1822 (1998).

[5] M. Lewenstein, A. Sanpera, V. Ahufinger, B. Damski, A. Sen, and U. Sen, Adv. Phys. 56, 243 (2007).

[6] H. Saito and M. Ueda, Phys. Rev. A 72023610 (2005).

[7] W. Zhang, D. L. Zhou, M.-S. Chang, M. S. Chapman, and L. You, Phys. Rev. Lett 95180403 (2005).

[8] J. Mur-Petit, M. Guilleumas, A. Polls, A. Sanpera, and M. Lewenstein, K. Bongs, and K. Sengstock, Phys. Rev. A 73, 013629 (2006).

[9] L. E. Sadler, J. M. Higbie, S. R. Leslie, M. Vengalattore, and D. M. Stamper-Kurn, Nature 443, 312 (2006).

[10] P. Maddaloni, M. Modugno, C. Fort, F. Minardi, and M. Inguscio, Phys. Rev. Lett. 85, 2413 (2000).

[11] M. Schellekens, R. Hoppeler, A. Perrin, J. Viana Gomes, D. Boiron, A. Aspect, and C. I. Westbrook, Science 310, 648 (2005).

[12] H.-J. Miesner, D. M. Stamper-Kurn, J. Stenger, S. Inouye, A. P. Chikkatur, and W. Ketterle, Phys. Rev. Lett. 82, 2228 (1999).

[13] D. S. Hall, M. R. Matthews, J. R. Ensher, C. E. Wieman, and E. A. Cornell, Phys. Rev. Lett. 81, 1539 (1998).

[14] H. J. Lewandowski, D. M. Harber, D. L. Whitaker, and E. A. Cornell, Phys. Rev. Lett. 88, 070403 (2002).

[15] P. Ziń, J. Chwedeńczuk, A. Veitia, K. Rzążewski, and M. Trippenbach, Phys. Rev. Lett. 94, 200401 (2005); J. Chwedeńczuk, P. Ziń, K. Rzążewski, and M. Trippenbach, ibid. 97, 170404 (2006).

[16] M. Moreno-Cardoner, J. Mur-Petit, M. Guilleumas, A. Polls, A. Sanpera, and M. Lewenstein, Phys. Rev. Lett. 99, 020404 (2007).

[17] W. Kohn, Phys. Rev. 123, 1242 (1961), S. Stringari, Phys. Rev. Lett. 77, 2360 (1996).

[18] W. Zhang and L. You, Phys. Rev. A 71, 025603 (2005).

[19] Cf. movie attached as EPAPS.

[20] J.F. Dobson, Phys. Rev. Lett. 73, 2244 (1994).

[21] J. J. Thorn, E. A. Schoene, T. Li, D. A. Steck, Phys. Rev. Lett. 100, 240407 (2008).

[22] S. Trotzky, P. Cheinet, S. Flling, M. Feld, U. Schnorrberger, A. M. Rey, A. Polkovnikov, E. A. Demler, M. D. Lukin, and I. Bloch, Science 319, 295 (2008). 